\title{
VARIA LEXXICOGRAPHICA GRAECA MANUSCRIPTA III: LEXICA BOTANICA
}

This paper examines the botanical lexica of mss. Escorialenses $Y$ III ro (280) f. $28 \mathrm{r}-\mathrm{v}$ and $\Sigma$ III $I_{7}$ (II6) ff. II $4 \mathrm{~V}-I_{1} I_{5}$, reidentifies the first of them and lists the significant variants of both. The complexity of this kind of lexicography is also considered.

Algunos de los caracteres generales de la lexicografía botánica griega de época bizantina han sido puestos de relieve, de forma excelente, por J. Stannard ${ }^{1}$ y esto nos dispensa de llevar a cabo cualquier breve introducción. Por otro lado, el fin que nos hemos propuesto en esta colección de notas ${ }^{2}$ no es otro que el de la identificación o descripción de las peculiaridades textuales de algunos mss. griegos conservados en España y ello es tarea que, aplicada al tema de estas páginas, tampoco necesita en demasía de cualquier introducción; baste con las precisiones más técnicas que, al final de este trabajo, hacemos al respecto.

El primer léxico que vamos a considerar es el contenido en Escorialensis Y III Io (280) f. $28^{\text {r.v }}$ que G. de Andrés ${ }^{8}$ describe como 〈Galeni $\rangle$

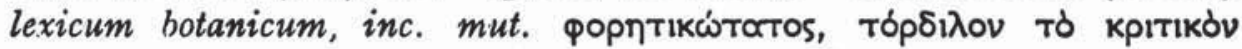
$\sigma \varepsilon \sigma \varepsilon \lambda \circ v$, remitiendo a $\mathrm{H}$. Diels ${ }^{4}$ y J. Fr. Boissonade ${ }^{5}$. Ahora bien,

1 "Byzantine botanical Lexicography", Episteme V, 1971, pp. 168-187.

2 Véase "Varia lexicographica graeca manuscripta I: de uocibus animalium", Habis, en prensa, y "Varia lexicographica graeca manuscripta II: sacra et profana", EMERITA 46, 1978, pp. 343-346.

- Catálogo de los códices griegos de la Real Biblioteca de El Escorial II, Madrid, 1965, p. I56: se trata de un chartaceus del siglo XV-XvI escrito por ocho manos diferentes y no identificadas.

- "Die Handschriften der antiken Aerzte" I, Abhandl. der Königl. Preussischen Akad. der. Wiss. Philos.-hist. Kl., Berlín, 1905-1906, p. I17.

- J. Fr. Boissonade, Anecdota Graeca e codicibus Regiis II, Paris, 1831, p. 407, 1. 9-408. Nota este autor en p. 394: $\mathrm{EE}$ codice 1310, p. 34. Illo usus est Cangius in Glossario; nam codices quas numeris 3118 et ${ }^{3} 8_{4}$ notavit, iidem huic sunt quem nunc tracto. In indice titulum libri Cangius inaccurate exhibuit». 
ni el primer autor menciona este ms. ni la EPMHNEIA חPOXEIPO $\Sigma$ ONOMATWN BOTANWN KAI ETEPWN EI $\triangle \omega N$ IATPIKWN AПO $\triangle I A \Phi O P \omega N$ BIBAIWN $\Sigma Y \wedge \wedge E \Gamma E I \Sigma A$ KATA A^ФABHTON, que es la obra editada por el segundo, ofrece otra cosa que un parecido algo lejano, ya que, de las veinticinco glosas (contando una marginal) que contiene el Escorialensis, tan sólo diez están recogidas en el texto que edita Boissonade y con algunas diferencias textuales. Conociendo la transmisión tan accidentada y fragmentaria de este tipo de literatura y su gestación, no cabe duda de que el parentesco es evidente, pero creemos que es posible hallar otros léxicos todavía más parecidos $y$, por tanto, más emparentados y, a la vez, pensamos que es posible corregir la autoría propuesta por De Andrés.

En primer lugar, a la vista de la edición que A. Delatte ${ }^{1}$ ha reali-

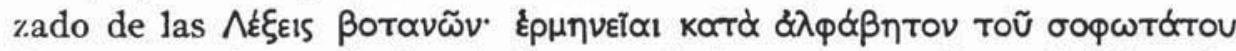
$\Gamma \alpha \lambda \eta$ voũ, podemos decir, sin lugar a dudas, que las glosas del f. $28^{r-v}$ del Y III ro (280) no están tomadas de este léxico, sino que proceden de uno atribuido al monje Neófito Prodrómeno, titulado Прóxєıpos

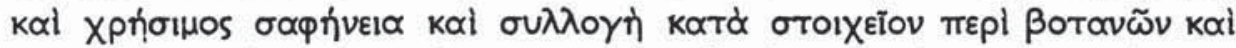

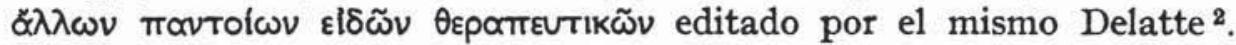
Efectivamente, comienza el Escorialensis casi al final del tratado y a

1 Anecdota Atheniensia et alia II. Textes grecs relatifs à l'histoire des sciences (Bibliothèque de la Fac. de Philosophie et Lettres de l'Univ. de Liège, LXXXVIII), Paris, 1939, pp. 385-393.

2 O. c., pp. 277-302. La edición está basada en los Parisini 2286 (del siglo xIv), 2256 (del siglo Xv) y Suppl. 684 (del siglo XvI) y el Berolinensis 166 (=Phillippicus 1570) que es del siglo xvi. Delatte los sigla respectivamente $\mathrm{R} Q \mathrm{P} B$ y menciona tres mss. más que no utiliza. Véase $\mathbf{M}$. Head Thomson, "Catalogue des manuscrits grecs de Paris contenant des traités anonymes de botanique, $R E G 46,1933$, pp. $334-338$, sobre otros léxicos similares contenidos en estos. mss. Aparte de una obra teológica, el monje Neófito, del monasterio de San Juan

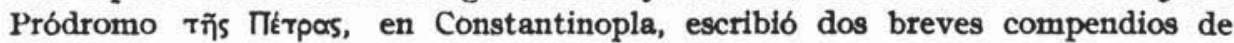
medicina. Efectivamente, los mss. le atribuyen la obrita que aquí analizamos (véase Diels, o. c., II, p. 68, no mencionando este Escorialensis) y un De dentium doloribus conservado en un ms. de Atenas. De su labor filosofica se ha conservado. un comentario autógrafo al Organon aristotélico (véase M. Vogel-V. Gardthausen, Die griechischen Schreiber des Mittelalters und der Renaissance, Leipzig, 1909 [hay reimpresión], p. 332) en el Vaticanus $\mathrm{g} r$. ror 8, pero lo más interesante es. que el Parisinus gr. 2286 y el Patavinus descubierto no ha mucho, códices ambos. de Dioscórides, fueron copiados, a lo que parece, por este mismo autor (véase E. Mioni, "Un ignoto Dioscoride miniato [Il codice greco 194 del Seminario di Padova]" en Libri e stampatori in Padova. Miscellanea di studi storici in onore di Mons. G. Bellini, Padua, 1959, pp. 345-376 y también, con láminas, la "rassegna. medican Convivium sanitatis 36, 1959, pp. 169-184). 


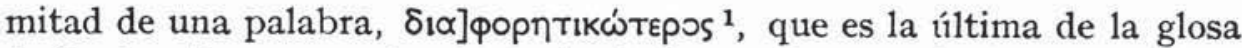

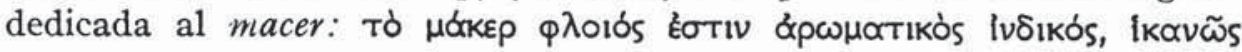

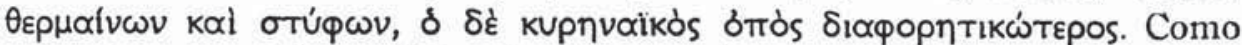

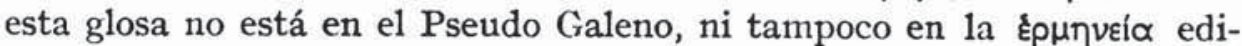

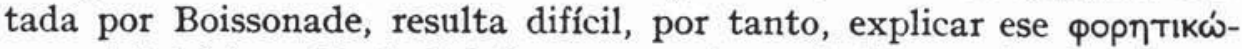

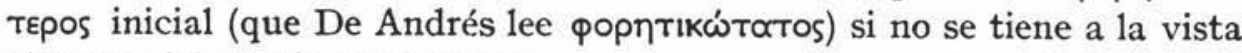
el texto del monje Prodrómeno.

Comparando el texto de Neófito con el del Escorialensis notaremos que todas las glosas de este último, y en el mismo orden, se encuentran en aquél, aunque unas cuantas del léxico atribuido al monje faltan del Y III ro (280). Damos a continuación el contenido del Escorialensis colocándolo frente (:) al texto impreso, si es que hay variación ${ }^{2}$, añadiendo entre paréntesis la página y línea de la edición y, entre corchetes, lo más interesante del aparato crítico que pueda ayudar a ubicar de forma aproximada las lecturas del texto que editamos (la numeración de las glosas, por supuesto, falta en el ms. y respetamos la ortografía de éste):

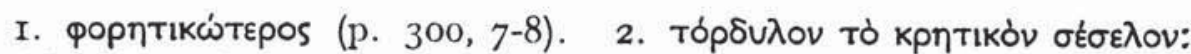

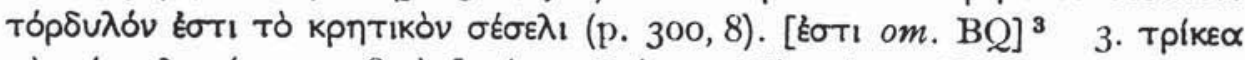

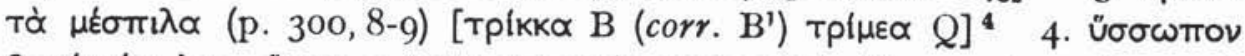

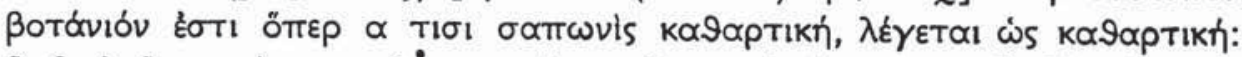

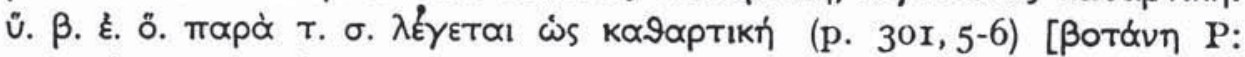

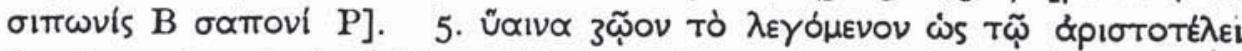

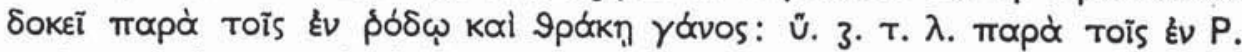

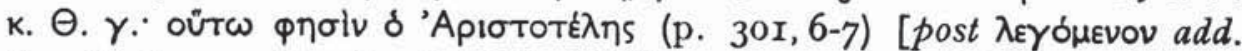

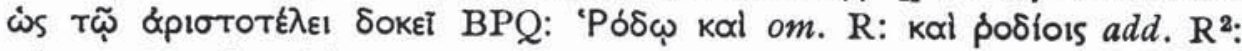

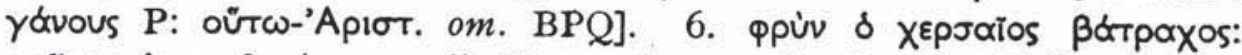

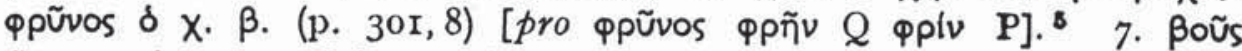

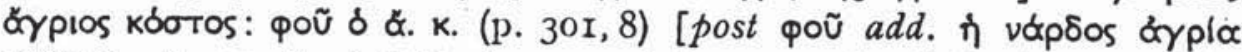

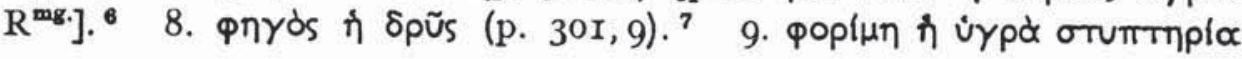

1 Véase Delatte, o. c., p. 300, 9.

2 No tomamos en cuenta la uglosa marginal del Escorialensis, aunque es de la misma mano (se trata de un añadido que se encuentra en los otros mss.:

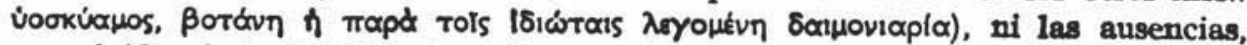
por olvido, de la inicial roja, faltas por itacismo, de cantidad, de geminadas y otras pequeñas confusiones de letras debidas, probablemente, a despiste del copista o ignorancia del vocabulario técnico empleado.

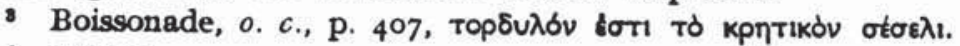

- Ibidem.

- O. c., p. 408 .

- O. c., p. 407 .

$\quad$ O. c., p. 408 . 


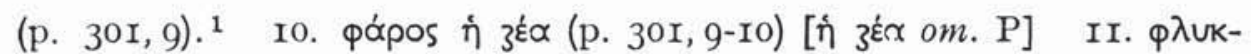

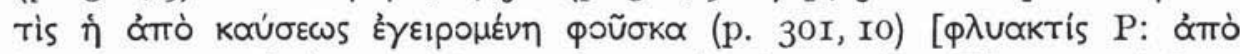

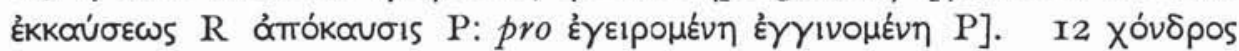

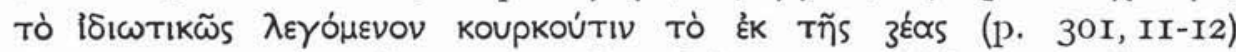

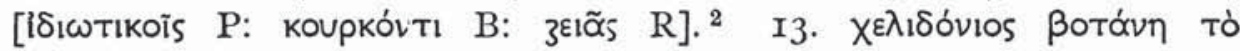

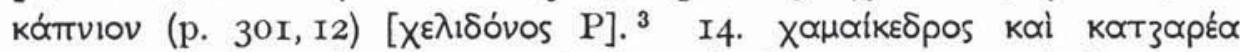

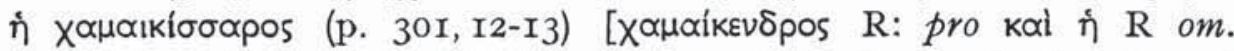

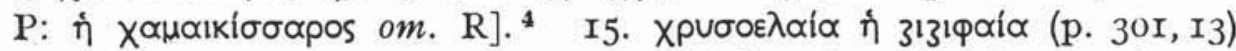

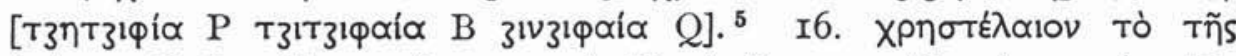

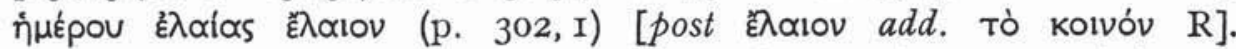

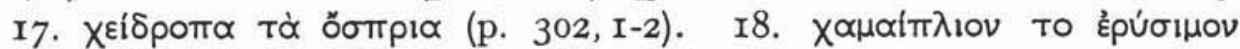

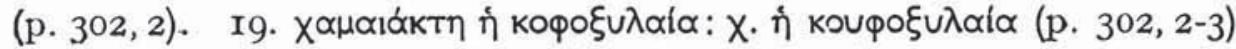

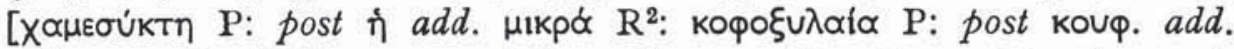

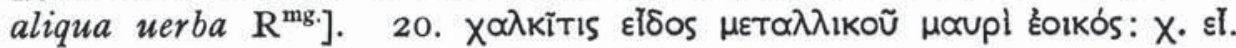

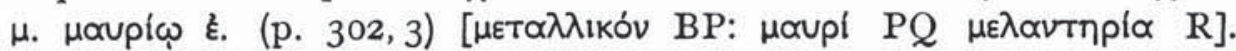

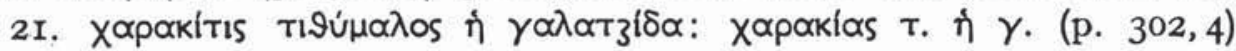

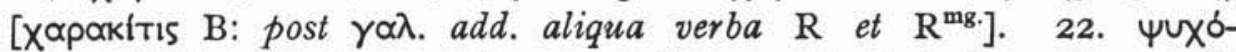

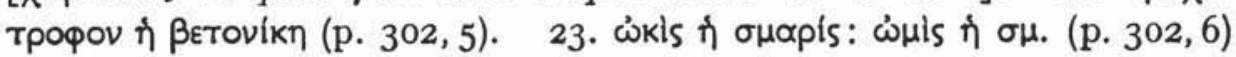

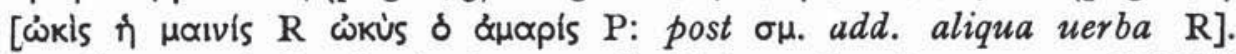

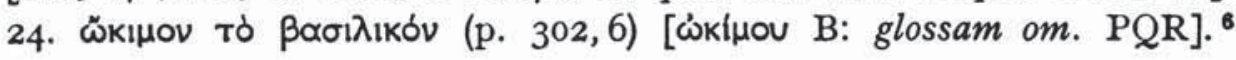

En resumidas cuentas, el Escorialensis parece ocupar una posición alejada de $\mathrm{R}$ y próxima a los otros tres, aunque, en ocasiones, va con unos $\mathrm{y}$, otras veces, con otros de este grupo. "BPQ - nos dice Delatte ? forment un groupe assez uni. $\mathrm{R}$ offre un texte tantôt plus court tantôt plus abondant, non seulement dans le développement des articles, mais aussi en égard à leur nombre». Copiosas notas - continúa- cubren los márgenes de $\mathrm{R}$ y añaden complementos o nuevos artículos $\mathrm{y}$ esto nos da una idea de la dificultosa maraña que forma la transmisión de estos pequeños léxicos copiados y recopiados ${ }^{8}$.

Ibidem.

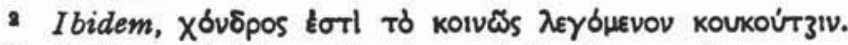

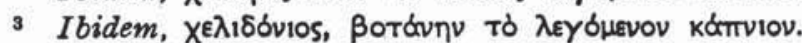

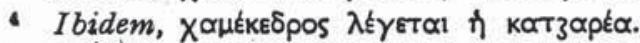

- Ibidem.

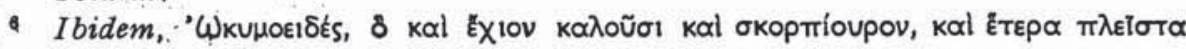

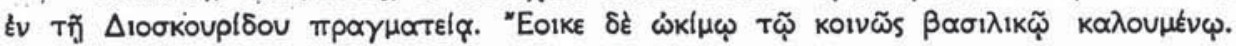

7 O. c., p. 277.

B Un detalle interesante es que el léxico contenido en y III 9 (332) ff. 214216' (véase G. de Andrés, o. c., pp. 227-229) es el mismo que el editado por Boissonade $y$, correctamente, así lo señala el catalogador, aunque no consigna su título que, como suele suceder, tiene alguna variante que otra en el ms. 
El segundo punto que vamos a tratar se refiere al $\Sigma$ III I7 (II6)

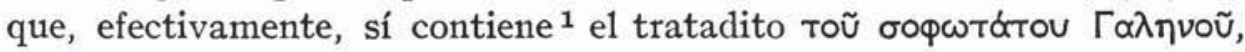
aunque esta atribución, en palabras de Delatte, «est naturellement sans valeur' ${ }^{2}$. Las cinco letras que recoge $(\alpha-\varepsilon)$ están llenas de faltas ${ }^{3}$ y muestran un texto acorde con el editado $(\mathrm{AB})^{4}$, aunque hay muchas glosas nuevas y algunos cambios de cierto interés que transcribimos a continuación:

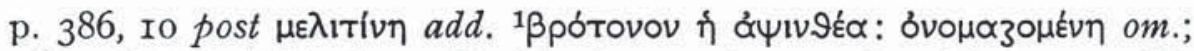

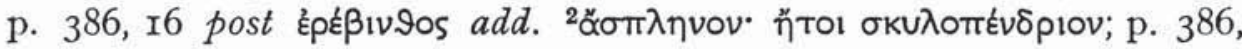

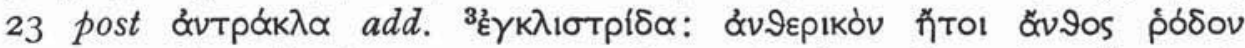

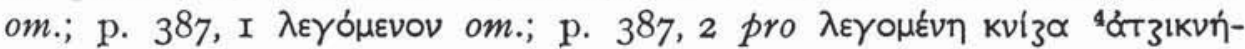

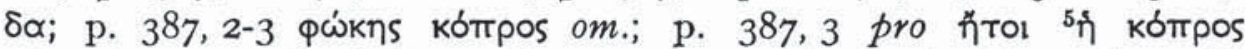

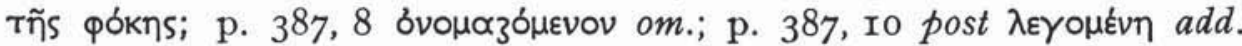

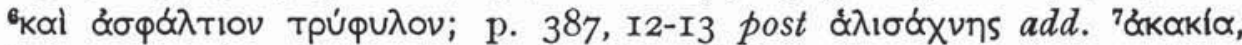

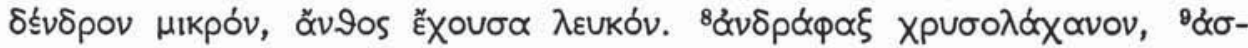

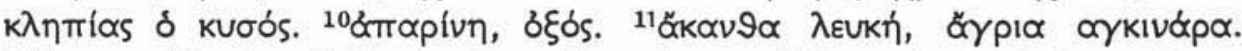

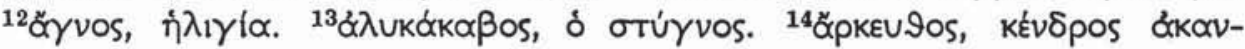

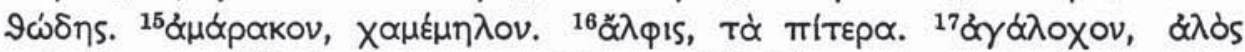

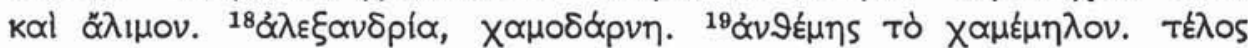

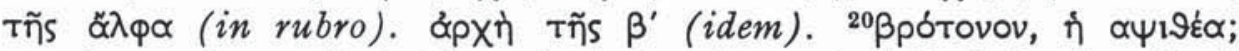
p. 387 , I5 pro то

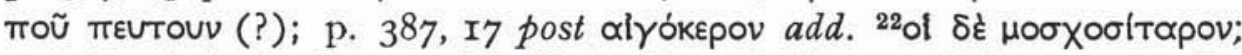

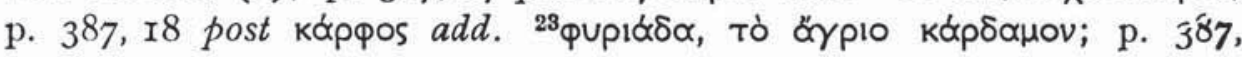

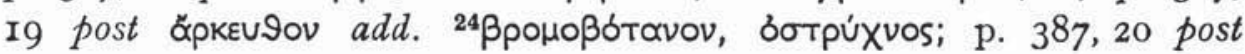

1 Véase A. Revilla, Catálogo de los códices griegos de la Biblioteca de El Escorial I, Madrid, I936, pp. 376-383 y Diels, o. c., p. II7. Se trata, esta vez también, de un chartaceus del siglo XV que en ff. II $4 \mathrm{~V}$-I I $5 \mathrm{~V}$ nos ofrece un texto que

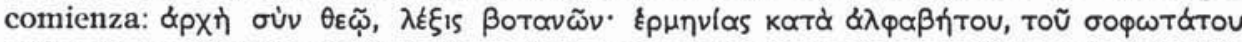
$\Gamma \propto \lambda \eta$ voũ. La edición de Delatte se basa en dos mss.: Parisinus I 883 , del siglo XIV (véase Thomson, o.c., p. 336: "texte apparenté à Dioscoride"), signado A, y Parisinus 2149 , del siglo XVI, signado B (véase Thomson, o. c., pp. 337-338). Otros mss. son los Parisini 2238 (del siglo xv), 2243 (a. 1339) y Coislinianus 335 (del siglo Xv) de los que Thomson habla, o. c., passim.

2 O. c., p. $3^{8} 5$.

3 Véase lo dicho en n. 2 de la p. 349 . Otros detalles sin importancia son igualmente pasados por alto, pero debemos señalar que el ms. en cuestión omite casi todos los fítor del texto impreso.

- Asi lo testimonian, por ejemplo, las peculiaridades de p. 386, I 8 (tal vez

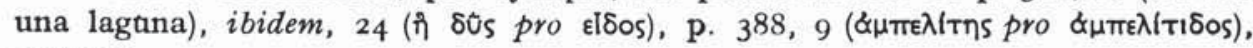
etcétera. 
$\lambda \varepsilon \gamma o ́ \mu \varepsilon v o s$ inc. falso littera $\gamma$ ( $\gamma \alpha \dot{\lambda} \lambda \alpha v o s$ pro $\beta \dot{\alpha} \lambda \alpha$ osos... $\gamma$ putía pro

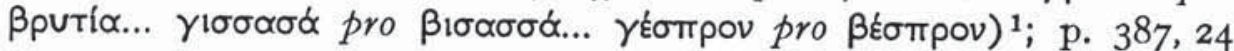

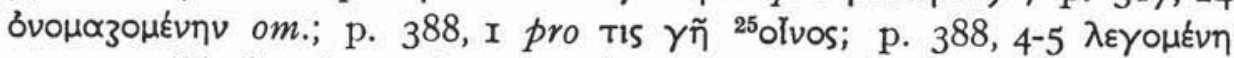

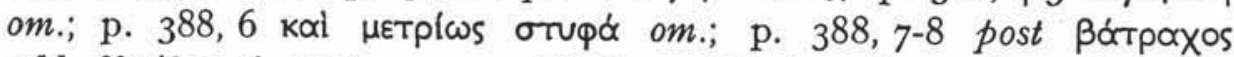

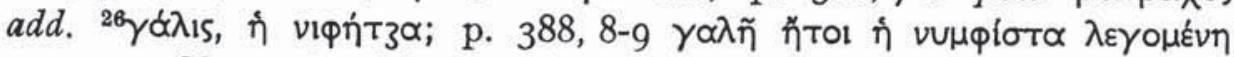

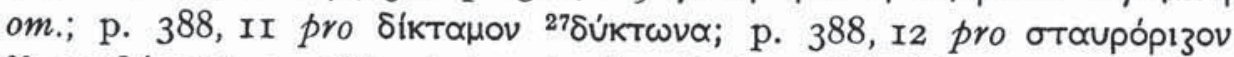

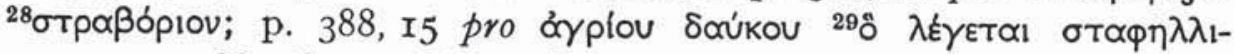

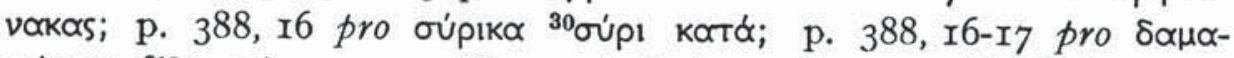

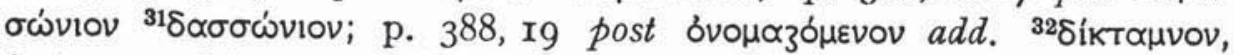

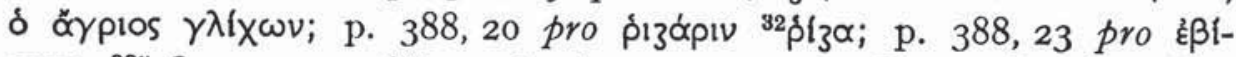

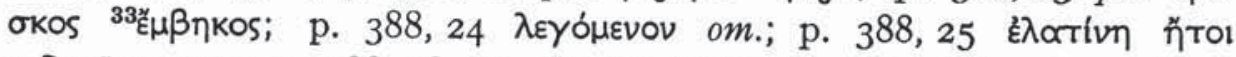

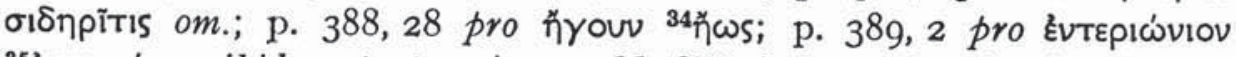

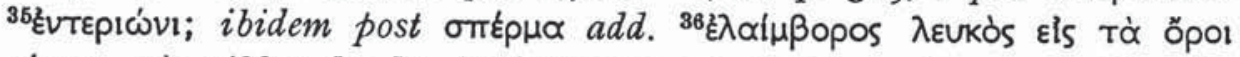

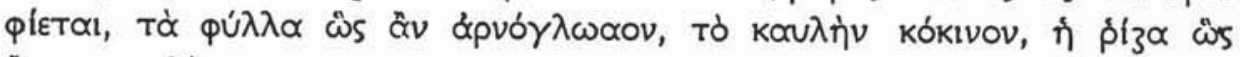

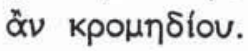

El material léxico aportado por este texto, con sus muchas y groseras faltas, está recogido, en su mayor parte, en los quince uglossaires de botanique" que Delatte editó, pero las variaciones son tan grandes, a veces, que convendría detenerse en este punto y tratar de arrojar un poco más de luz sobre el funcionamiento de este tipo de lexicografía. Consideremos, por ejemplo, una de las "novedades" de este $\Sigma$ III I7

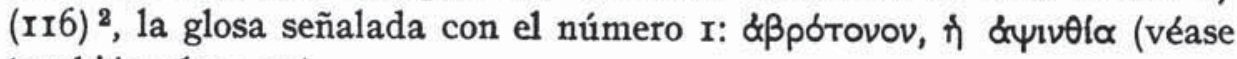
también glosa 20).

Se refiere esta glosa a la artemisia arborecens, el abrótano ${ }^{3}$, que aparece en Teofrasto, Historia Plantarum VI 7,3 y no se halla recogida exactamente igual por Delatte en los otros léxicos. Así, encontramos

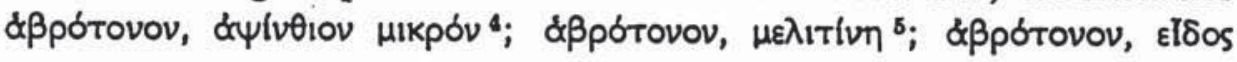

1 La confusión (acabar la letra $\beta$ y empezar la colección de las glosas comenzadas por $\gamma$ antes de tiempo) es testimonio de la poca pericia del copista en estos temas botánicos y disculpa sus otros muchos errores.

2 Citamos de la obra de Delatte únicamente la página, sin especificar línea ni léxico concreto.

8 Véase A. Carnoy, Dictionnaire étymologique des noms grecs de plantes, Lovaina, 1959, p. I y, en otro aspecto, J. Murr, Die Pflanzenwelt in der griechische Mythologie, Innsbruck, I890, pp. 200-201; también B. Langkavel, Botanik der späteren Griechen vom dritten bis dreizehnten Jahrhunderte, Berlin, 1866, pp. 72-73.

- $O$. c., pp. 3 I9 y 3r5.

- Ibidem, pp. 307, 319 y 382 . 


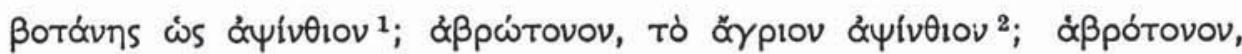

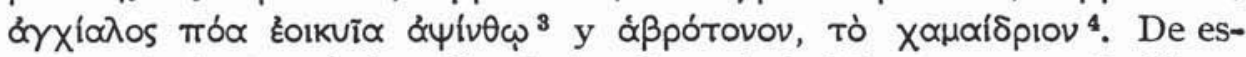

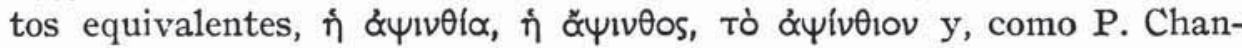
traine $^{5}$ añade, $\delta$ ŏ $\psi \imath \bullet \theta$ os, son los nombres que designan la artemisia absinthium (nuestra 'absenta' o, mejor, 'ajenjo') ${ }^{6}$ o bien la artemisia monosperma ${ }^{7}$. De la $\mu \varepsilon \lambda{ }_{i t i v \eta}$ nada se nos dice por parte de Carnoy,

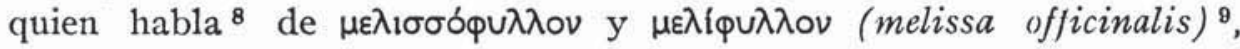

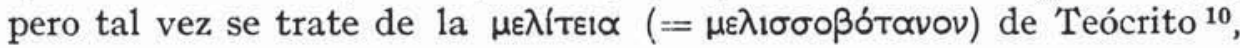

1 Ibidem, p. $34 \mathrm{I}$.

2 Ibidem, p. 342.

3 Ibidem, p. 373.

- Ibidem, p. 378 .

- Dictionnaire étymologique de la langue grecque. Histoire des mots, París, I 968 (fasc. r), p. I52.

- En español es un galicismo innecesario «absenta" (véase M. Seco, Diccionario de dudas $y$ dificultades de la lengua española, Madrid, 1970 [2. ${ }^{\text {a }}$ reimpr. de la $3^{\mathbf{a}}$ ed. 7, p. 7), teniendo nuestra lengua un vocablo, aajenjow, que proviene del griego a través del latín (véase J. Corominas, Breve Diccionario etimológico de la lengua castellana, Madrid, $1967_{2}$, p. 34). La adopción de la palabra francesa se explica, tal vez, por la abundancia de esta planta y su uso en nuestro vecino país. "Hállase otra especie de Absinto, la cual nace copiosamente en la Francia vezina a los Alpes, y se llama en aquellas partes Santonico, tomando apellido de la tierra que la produce, llamada Santonia. Parecese aquesta planta al absintio, aunque no engendra tanta simiente. Es algun tanto amarga, y tiene la mesma facultad que el Seriphio" (Pedacio Dioscorides Anazarbeo, Acerca de la materia medicinal, $y$ de los venenos mortiferos, Traduzido de la lengua Griega... por el Doctor Andres de Laguna..., Salamanca, 1566, p. 282.

7 Véase H. G. Liddell-R. Scott-H. S. Jones, A Greek-English Lexicon, Oxford, I968 (7. reimpr. de la $9 .^{\text {a }}$ ed.), s. $u$.

B O. c. pp. 175 y 176 .

- Véase Dioscórides, libro III ro4; citamos por la edición de M. Wellmann, Pedanii Dioscuridis Anazarbei de materia medica libri quinque, 3 vols., Berlín, 1907 .

${ }^{10}$ Id. IV 25 y V r 3 . Al primer pasaje anota A. J. F. Gow, Theocritus. Edited with a Translation and Commentary II, Cambridge Univ. Press, 1965 (reimpr. de la $2 .^{\mathrm{a}}$ ed.), pp. $82-83$ : " $\Sigma$ assert that it has a broad leaf like a lentil... that it resembles oivinßpiov (mentha aquatica)... that it is commonly called

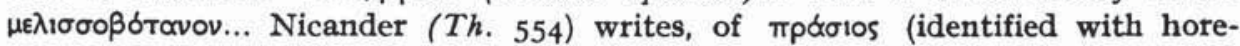

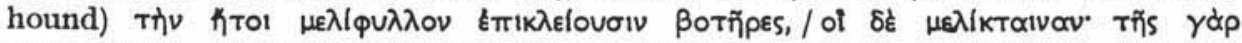

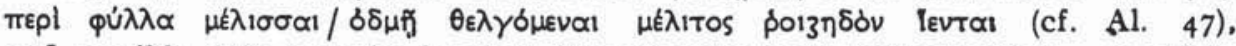

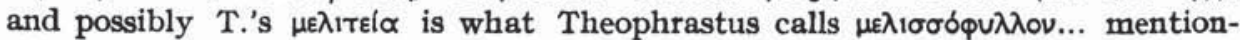
ed... next to mpdoriov and $k \delta v_{z} \alpha . .$. and according to Diosc. 3. Io4 was sometimes called $\mu \varepsilon \lambda i \tau \tau \alpha i o v, \mu \varepsilon \lambda i \tau \tau \alpha I v \alpha$ and $\mu \varepsilon \lambda i \varphi v \lambda \lambda o^{\prime}$. Véase A. S. F. Gow-A. F. Scholfield, Nicander. The Poems and poetical Fragments. Edited with a Translation and Notes, Cambridge Univ. Press, 1953, pp. I8 I y 234 para la diferenciación 
aunque esto no es seguro; de la planta denominada $\chi \propto \mu \alpha i \delta$ pus (teucrium chamaedrys $^{1}$ ), finalmente, nos ponen en antecedentes Carnoy ${ }^{2}$ y A. Huxley-W. Taylor ${ }^{3}$. Las tres plantas últimas se relacionan entre sí, fuera de otros detalles técnicos, en que se utilizan todas ellas en la fabri-

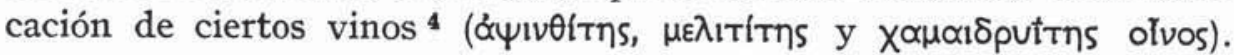
Aparte del aßßótovov ǻppev (artemisia fragans) y del \&ßpótovov $\theta \tilde{\eta} \lambda u$ (santolina chamaecyparissus) ${ }^{5}$ no aparece en el diccionario de L. S. J.

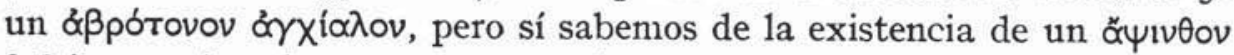

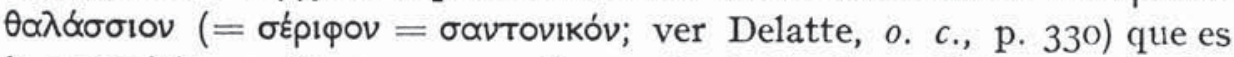

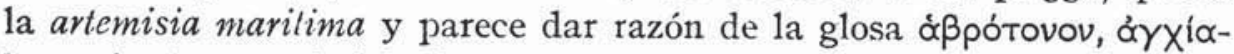

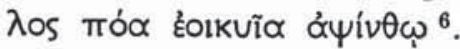

En fin, el haz de referencias que se cruza y se entrecruza en estas glosas se repite a lo largo y ancho de todos los léxicos suscitando curiosos problemas que interesan al lexicógrafo. No obstante, los numerosos errores de todos estos mss., su fragmentario origen y el dudoso valor que poseen, invitan con mucha frecuencia a descartar todo estudio de ellos; sin embargo, lo repetimos, hay denominaciones en algunos que

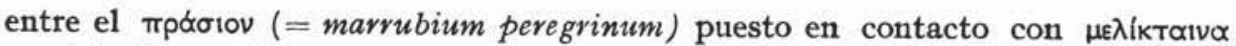

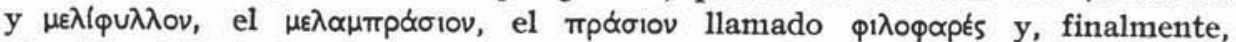
el mpđoov (Th. 879) que es tal vez la melissa officinalis. Véase, en general, A. L. A. Fée, Flore de Théocrite et des autres bucoliques grecs, Paris, I832 (hay reimpresión) y K. Lembach, Die Pflanzen bei Theokrit, Heidelberg, I970.

1 Dioscórides V $4 \mathrm{I}$; sobre la primera parte del nombre de la planta recor-

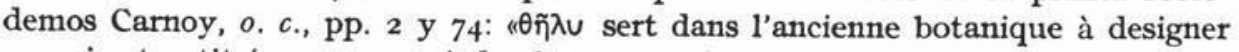
ce qui est petit (comme $\chi \propto \mu \alpha$ d de chamaecyparissos)". En Dioscórides III 97 aparece como semejante al teúkpiov y en III II I como igual a la planta denomi-

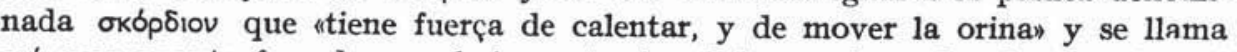
asi "por quanto fregadas sus hojas entre los dedos expiran olor de ajo, al qual llaman los griegos Scorodon", según dice Laguna en su traducción, p. 344. El TEúkpiov (Carnoy, o. c., p. 260) es el teucrium flauum, la kgermandrée mediterranéenne».

2 O. c., p. 75 .

3 Flowers of Greece and the Aegean, Londres, 1977, p. 123.

- Dioscórides V $4 \mathrm{I}$; véase C. Daremberg-E. Saglio-E. Pottier, Dictionnaire des antiquites grecques et romaines V, Paris, 1919, p. 920 y, en general, Cl. Seltmann, Wine in the Ancient World, Londres, 1957.

- Véase Carnoy, o. c., p. 2.

- La idea que Dioscórides tiene, en resumen, de esta cuestión es que el abrótano hembra se parece al serifio y el macho al absintio; de este último hay, en general, cuatro clases: común (parecido a la artemisia), póntico o romano, marino (= serifio) y santónico. Para usos de esta planta véase, además, V. Gazza, "Prescrizioni mediche nei papiri dell'Egitto greco-romano II. Le droghe medrcinali», Aegyptus 36, 1956, p. 79 . 
no son frecuentes; en otros, los menos, textos de cierto valor ${ }^{1} \mathrm{y}$, en ciertas obras del género, las más destacadas, menciones de gran interés.

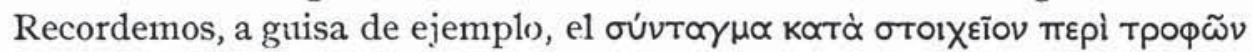
$\delta u v \alpha ́ \alpha \varepsilon \omega v$ de Simeón Seth ${ }^{2}$ donde se citan, algunas de ellas por primera

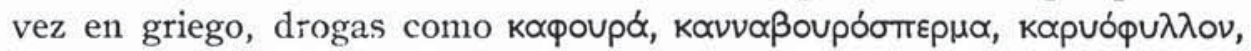

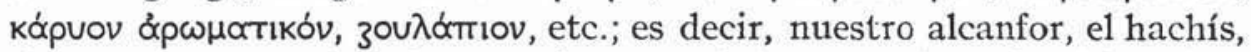
clavo, nuez moscada y los reconfortantes julepes. La botánica bizantina, cierto es, "cannot be understood apart from its scholary lexicographical orientation", como Stannard ${ }^{3}$ asevera, y las notas ar!ui reunidas aspiran a ser útiles a los estudiosos del tema desde ambos puntos de vista.

\section{Antonio Bravo García}

\footnotetext{
1 Véase, por ejemplo, M. Head Thomson, Textes grecs inédits relatifs aux plantes, Paris, 1955.

2 Edición de B. Langkavel, Leipzig, I868. Delatte, o. c., pp. I-I27, edita

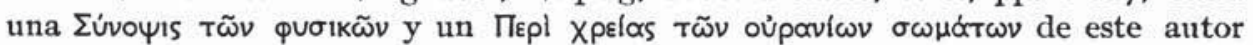
sobre el que puede verse, con estos y otros detalles, el artículo dedicado a él por G. Sarton, Introduction to the History of Science I, Washington D. C., Carnegie Institution, $1927 \mathrm{~J}$, en general, H. Hunger, Die hochsprachliche profane Literatur der Byzantiner II. Philologie, Profandichtung, Musik, Mathematik und Astronomie, Naturwissenschaften, Medizin, Kriegswissenschaft, Rechtsliteratur (mit Bei-

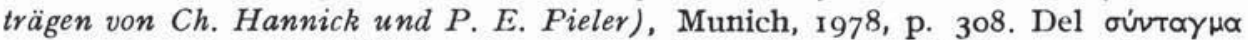
hay un Fscorialensis ( $\Psi$ IV 27 [5OI] ff. $24-82 a^{v}$, del siglo XIII) que no fue considerado por Langkavel ni G. Helmreich, Handsschriftiche Studien zu Symeon Seth (Programm des K. humanistischen Gymnasiums in Ansbach), Ansbach, 1913.

s O. c., p. 169.
} 